\title{
Managing the Primary Care of the Future: Evidence, Gaps, and Desiderata from Italy
}

\author{
Federica Segato \\ Politecnico di Milano \\ Cristina Masella \\ Politecnico di Milano
}

The demand for care is changing toward more primary care oriented needs. Hence, General Practitioners (GPs) need to redesign their practice, but they do not necessarily know how.

The aim of this paper is to understand which competences GPs need in the new healthcare settings. Referring to the competences identified by Bohmer (2010), we performed 16 in-depth interviews with GPs, nurses and assistants working in Italian primary care organizations. The results showed that most competences are at an immature stage of development and some are a prerogative for nurses and assistants. These findings call for actions by the policy makers.

\section{INTRODUCTION}

Intense debate is ongoing about how to make the primary care systems able to respond to challenges such as complying with an increasing demand from chronic patients, by simultaneously containing the costs and enhancing the quality of care (Saltman et al., 2005; Morrin, 2013; Truglio et al., 2012; Chouvarda et al., 2015, Lenander et al., 2015; Bienkowska-Gibbs et al., 2015). To meet these expectations, General Practitioner (GPs) should relook profoundly at their practice, from being reactive to the patients' self-activation, to managing the patients in a proactive, bundled and shared way (Bohmer, 2010).

Being "proactive" means to be able to keep a significant portion of patients under control, by preventing disease occurrence and unnecessary complications or comorbidities (Morrissey, 2013). Thus, the focus of general practitioners shifts from curing ill people, to taking care of an entire population (i.e. population health; Tallia and Howard, 2012; Truglio et al., 2012).

Being "bundled" implies for primary care practitioners to focus on the patients' care pathway in a medium to long time span, rather than on isolated acute incidents. To support this change, primary care financing should shift from pay-for-performance to bundled payments, which could reward the quality of the pathway instead of the volume of care provided to the patients (Baron, 2012; Morrissey, 2013).

Last, being "shared" refers to the delivery of care in a multidisciplinary endeavor, by enacting solutions which include physicians, nurses, therapists, social workers, educators and empowered patients (Bohmer, 2010). As such, the general practitioners' role is redefined, so that the physician no longer sees 
all the patients, but he/she acts as a leader of a primary care-based team (Margolius and Bodenheimer, 2010).

In this process of transformation, GPs are moving into uncharted territory (Scherger, 2007). For them, "it may seem, at times, that life is being turned upside down" (p. 8; Morissey et al., 2014). The myth of the "lone physician" (Saba et al., 2012), who was typified as a practitioner working in autonomy and controlling the relationship with the patients, is questioned by an emerging paradigm characterized by teamwork, group practice, technology-mediated interactions for managing care (Busari, 2012). This is a considerable cultural shift (Tallia and Howard, 2012), which requires GPs to go beyond pure clinical practice, toward holding a managerial role in multi professional primary care organizations, despite they are not necessarily trained for this (Barzdins and Barzdins, 2013; Busari et al., 2011; Fitzsimmons and White, 1997).

In this respect, while financial and organizational solutions for primary care are object of study and debate and mandated through policy acts, the competences needed by General Practitioners in these new organizations are not addressed systematically. The case of Italy is exemplary in this sense: several reforming actions, the last of whom is a national law in 2012 (D.L. 13/09/2012, n.158), encouraged the formation of primary care organizations, by providing also economic incentives for GPs to encourage the shift toward proactive, bundled and shared care. As it happens oftentimes, GPs were left free on how to organize processes, to structure relationships and to manage financial assets, to make these new organizational forms work (Segato and Masella, 2015). However, their preparedness in terms of skills and competences to manage these new arrangements were not object of reflection, therefore jeopardizing their success (Hall et al., 2015; Bohmer, 2010; Lane, 1998; Essex, 1981). In turn, except for few and theoretical contributions (e.g. Hart et al., 2015; Blumenthal et al., 2012; Bohmer, 2010; Homer and Baron, 2010; Scherger, 2007), the literature is essentially silent on the competences that GPs would need to manage these new organizational arrangements.

In this paper, we use Italy as a setting to explore which competences GPs need to manage the new primary care organizations. To this end, we proceeded in two steps. First, we defined a framework to outline the managerial skills for General Practitioners within the reorganization of primary care. Second, we conducted 16 in-depth interviews to General Practitioners, nurses and assistants working in Italian primary care organizations. During the interviews, by applying the theoretical framework to the Italian context, we investigated how the new organizations are currently managed, and which criticalities or open issues arise. Last, implications for healthcare managers and policy makers are explored.

\section{THEORETICAL FRAMEWORK: MANAGERIAL COMPETENCES FOR GENERAL PRACTITIONERS}

In the literature streams about the competences that GPs need to sustain the wave of reform of primary care, only marginal attention is devoted to organizational or managerial contents, while the contributions on clinical issues are prevalent, especially about treating chronic conditions (Rubin et al., 2015; Loeb et al., 2015) and mental diseases (Cohen et al. 2015). This is consistent with the arising necessity that primary care bears the treatment of chronic and long-term conditions, by delivering comprehensive care, from prevention and early diagnosis, to periodical follow-ups and coordination of specialist interventions, and home-based end-of-life care (Rubin et al., 2015). Despite clinical issues fall outside the scope of this article, it descends from the expectations expressed in the contributions mentioned above that a GP working alone cannot offer proactive, comprehensive and coordinated care to people affected by long-term and chronic conditions; to be effective, he/she needs an integrated and multidisciplinary organization in support. Consequently, GPs are now pushed to operate in practices with increased size and professional diversity (from nurses, to assistants, to social workers and psychologists, etc.).

However, few articles deal with the competences needed to general practitioners to manage these new organizational settings. A contribution by Bohmer (2010), which represents the most comprehensive framework recently published on managerial competences for GPs, provides the basis for our discourse. 
In his article, he highlighted eight essential skills for physicians to manage the new primary care organizations: operations design, data management, human resources management, leadership, negotiation and conflict resolution, team management, innovation and performance evaluation, financial control, and capital allocation. Table 1 provides an overview of these competences.

\section{TABLE 1 \\ SUMMARY OF MANAGERIAL COMPETENCES FOR GPS [ADAPTED FROM BOHMER, 2010].}

\begin{tabular}{|l|l|}
\hline \multicolumn{1}{|c|}{ Competence } & \multicolumn{1}{c|}{ Description } \\
\hline Operations design & - designing protocols for clinical issues, but also for medical \\
workflows; \\
- task allocation; \\
- information flows requirements; \\
- routine for professionals' coordination.
\end{tabular}

In the light of the Bohmer's framework, two issues arise: first, the questionable width of this framework; second, the attribution of all these competences to the GPs.

First, about the width of the framework, as Bohmer and other authors argue (e.g. Blumenthal et al., 2012; Homer and Baron, 2010), the list of competences included in the framework is daunting for practitioners. In turn, some authors focus only on few of them, such as teamwork (e.g. Eyre and Durning, 2015) or human resources management (e.g. Homer and Baron, 2010). Therefore, whether all the skills identified by Bohmer are useful or not for GPs to manage the new primary care organizations is not clear. Moreover, these contributions are mostly conceptual and theoretical, while we do not know much about the actual experience of practitioners in managing the new primary care organizations. Therefore, while there is consensus on the fact that the absence of these skills could jeopardize the achievement of successful and sustainable improvements in primary care (Hart et al., 2015), no consistent investigation has been done on whether these skills are all useful for GPs in the practice or not.

Second, Bohmer argues that the eight skills are necessary for General Practitioners; however, according to the increasing size and differentiation of professionals in primary care organizations, these 
competences could actually be diffused in the organization, instead of being prerogative for the GP. This possibility has not received systematic attention in the literature so far.

To address these two issues, in this study we apply the theoretical framework developed by Bohmer in 2010 to primary care organizations in Italy, in order to understand which competences are actually needed to manage them and who holds these competences.

\section{MATERIALS AND METHODS}

The limited evidence available on the managerial competences needed for Italian General Practitioners in the new organizational forms justifies the adoption of an exploratory qualitative approach (Strauss and Corbin, 1998), based on observations and interviews. The interviewees' selection was made through a training course, held in June 2016 in Parma (Italy), which involved 33 Italian General Practitioners, belonging to different primary care organizations. Within this course, one of the authors chaired a session about the managerial competences for General Practitioners. During the session, she presented and discussed the framework in Table 1. After the lecture, the participants discussed about it. The discussion was useful to detect critical areas (such as gaps in competences and training of GPs), to be investigated further during face-to-face interviews. After the training event, eight GPs accepted to give access to their organizations for an in-depth investigation.

The aim of the interviews was to understand how GPs manage the two mostly diffused organizational forms for collaborative primary care in Italy, i.e. GPs' networks and groups. In the former, GPs receive financial incentives to share the electronic patients' records with some colleagues working geographically close; in this way, if the patients need assistance when their GP is absent, they can turn to other GPs, who have access to the electronic record and can act accordingly. In groups, GPs receive incentives not only to share the electronic patients' record, but also to work in the same location and hire support staff, such as nurses or assistants. In Table 2, we report the interviewees according to the type of practice they are part of. We reached the interviewees in their own environments, and performed interviews with both GPs and other professionals working in the organizations. Overall, we interviewed practitioners belonging to 4 different groups and 3 different networks. Interviews lasted 1 to 2,5 hours; they were recorded, transcribed verbatim and validated by the interviewees. Moreover, field notes were collected by the researchers. Interviews and observation took place from September to December 2016.

TABLE 2

\section{OVERVIEW OF INTERVIEWEES}

\begin{tabular}{|l|l|l|}
\hline & \multicolumn{1}{|c|}{ Working in Networks } & \multicolumn{1}{c|}{ Working in Groups } \\
\hline GPS & 4 & 4 \\
\hline Nurses/Assistants & 3 & 5 \\
\hline TOTAL & 7 & 9 \\
\hline
\end{tabular}

Interviews transcripts, field notes, and the reports of the focus groups produced by participants were analyzed through process coding (Corbin and Strauss, 2014). For content analysis, two authors proceeded separately, by coding emerging themes and met periodically to seek convergence over codes and categories. The data coding was supported by N-Vivo 10 . 


\section{RESULTS}

In this section, we report the results gathered from the interviews, and we organize them following the framework provided in Table 1. The interviews showed that the competences needed to run different primary care organizations (i.e. networks and groups) are basically the same. As such, we treat them together in this section.

\section{Operations Design}

Operations include both front and back-office processes. Front office processes consist of managing the relationship with the patients (i.e. managing referrals and appointments, and delivering clinical services). In the cases, nurses and administrative staff are the interface with the patient: they collect necessities, set appointments and handle referrals. Often nurses also perform a triage, and decide whether to treat the patient (e.g. measuring blood pressure, perform a medication), set an appointment with the GP, or send the patient to the hospital. Thus, GPs meet only the patients that really need a medical examination. This allows the GPs to stay focused on clinical issues (a nurse affirmed: "We have a lot of patients who come just to ask for information or to collect referrals, or for a routine check of the blood pressure: GPs don't see them anymore"). For clinical activities, almost all GPs reported that they are working to develop (or they already use) shared protocols and guidelines to uniform the patients' treatment and make it aligned to international clinical guidelines.

Back office activities consist of managing the relationships with suppliers (especially pharmaceutics and disposable equipment such as gloves or syringes) and of managing administrative/legislative requirements. Most organizations have nominated internal referrals (GPs, nurses or assistants), that keep track of deadlines and legislative updates, and report periodically to the other members of the organization about the situation or about additional requirements to be met (e.g. mandatory safety courses, new hygienic requirements, etc.). In this respect, being together allows to make the most of the individuals' specific expertise, both for clinical (a GP affirmed that: "If I need a second opinion on a patient of mine, I just have to enter the door next to my office and ask her opinion") and technical/administrative duties (a GP said: "according to their personal interests, people here volunteer for being the appointed assistant who takes care of the relationships with suppliers, the appointed for technical and information system issues, for financial issues and payments... we also have a responsible for coffees and party!").

\section{Data Management}

The data management in the primary care organizations analyzed in this study consists only in collecting and updating data on the GPs' agendas, on the administrative information systems, and on the patients' medical records. Nurses and assistants manage agendas and administrative data flows, which should be sent to the local health authority for appropriateness control. They are expert of the information system and of its functionalities. Data on the patients' medical records are instead uploaded and updated by the GPs. However, nurses and assistants often support the GPs in these activities. However, no data are currently collected about the organizations' performances in terms of clinical or financial outcomes.

\section{Human Resources Management}

The interviewees argued that the differentiation and increased number of professionals in the organizations makes managing people and relationships among them necessary; however, Italian primary care organizations still fall short in managing human resources strategically.

The interviewees reported that GPs hold continuous relationships with nurses and assistants, to keep commitment, to share problems and to agree on clinical and organizational procedures. In some cases, this happens through formal and periodic meetings; other organizations keep it more informal. Nevertheless, no practices such as structured recruiting and training are currently in place. Recruiting is unstructured and informal, and no specific training is conducted for assistants and nurses, to align them on the primary care practice. In this respect, a nurse reported that: "They told me: 'this is your desk'. But no one told me 
what was my duty here as a nurse, what I should have been doing all the time". An administrative assistant argued: "The first day here I was upset. You know, I had been working in the commercial office of a small private company until the previous day, so here I hardly understand the language they used! All those medical terms... I wasn't confident with this. So I remember I came home that very day and I started to look for a training course for formal caregivers. That's totally different from my job, I am just a secretary... but at least I could learn something, some right wording, some concepts".

About performance management, most GPs organize peer-to-peer clinical audits. The performances of nurses, assistants and other professionals are not currently monitored and assessed.

\section{Leadership, Negotiation and Conflict Resolution}

The interviewees argued that organizations need a leader competent on both economic and clinical issues, capable to drag the colleagues toward a mindset and cultural shift. However, most organizations are hinged upon informal leaders. The interviewees assessed that leadership is diffused, democratic and peer-to-peer. The leaders' duty is to maintain the formal relationship with institutions and be a point of referral in case criticalities within the organization emerge.

The interviews showed that day-by-day negotiation and conflict resolution are not handled by the GPs. In most cases, nurses and assistants mediate the relationships occurring in the organizations: they become the recipients and solvers of conflictual situations. For example, different GPs have different requirements on how assistants should manage their agendas and on how much nurses should be autonomous in treating their patients. Assistants and nurses work hard to negotiate the organizational rules and to make them more effective and efficient ("we worked hard to pass a clear message to the GPs: we can't treat each one of them differently! Making exceptions creates overloads and disorder, and this penalizes the other GPs and the patients"). Finding the correct balance and avoiding conflicts is part of the nurses and assistants' job ("we are a filter for discontents and disagreements").

\section{Team Management}

About working in team, the organizations differ for the breadth of their vision. As said above, GPs in networks receive incentives for connecting the electronic medical records of patients between different GPs. However, one interviewee argued that he takes advantage of being part of a network just to get coverage in case of holidays; in turn, others take advantage of the connection to define common practices and guidelines to improve access and quality of care for patients. Conversely, groups receive incentives to hire nurses or administrative staff (e.g. secretaries). However, some groups set the organization in order to exploit team practices; others do not actually work in team; a nurse reported that 'They [the GPs] don't actually know what I am doing here all the time".

In the cases where a real collaboration is in place, GPs are focused on clinical practice: they leave administrative duties to administrative staff, and share the organization of care with nurses ("working in teams, where our duties can be shared with nurses and assistants, allows to be proactive: we are not just waiting for the patients to come when they feel they need it - as we did in the past. Now we get in touch with the patients; we collect information on their health status periodically; we try to prevent diseases and complications...things like these"). Nurses are in charge to organize prevention or screening campaigns, in accordance with the GPs.

\section{Innovation and Performance Evaluation}

GPs are engaged in attempts to innovate clinical practice and to evaluate clinical performances. The interviewees reported that within the new organizational forms they recovered time to define care protocols, to be updated on specific diseases, to conduct clinical audits, to take part in innovative projects. Moreover, working collaboratively provides a stimulus for the innovation of clinical practice ("since when we became a group, we all grew up as practitioners: for example, we are doing very interesting things for people with diabetes; we also published a study recently (...). I would have not been able to do all this alone"). Clinical audits are conducted to monitor clinical performances, but they are mostly kept informal. No consistent effort is made on managing the organizational performances yet. 


\section{Financial Control and Capital Allocation}

About financial control, interviewees mainly mentioned activities such as management of invoices or other financial issues, and monitoring administrative and legal requirements (e.g. periodical maintenance of devices). Capital allocation - as the capability to use financial resources to make investments - is not applied in a strategic way, but just in reaction to emerging necessities (e.g. replacing broken equipment). Interviewees argued that financial control is not perceived as strategic activity: some organizations manage it internally, while others outsource the management of financial resources to external accountants or appointed organizations. The choice is driven by presence or absence of a person in the organization who is keen to care for it ("before becoming a GP, I was a bookkeeper and I am son of bookkeepers...so it's in my blood').

\section{DISCUSSION}

The aim of this study was to understand - beyond the theory - which competences GPs need to manage the new primary care organizations, within the specific context of Italy. With respect to the comprehensive framework provided by Bohmer, we intended to investigate: first, whether all the competences included in the framework are useful for GPs in the practice; second, whether these competences are a prerogative for GPs only. In this respect, two main areas for discussion emerged from the interviews, and they are reported in the following sections.

\section{Applying the Framework to Italy}

All the competences included in the framework by Bohmer (2010) emerged as useful to manage the Italian primary care organizations. However, some of them are still at an immature stage of development.

On the one hand, this could be due to cultural factors. For example, the interviews showed that maturity on financial control and capital allocation has still not been reached in Italian primary care organizations: many GPs still prefer to outsource the management of financial resources to external accountants. Competences such as financial control or capital allocation are in the mindset of GPs in insurance-bases systems. On the contrary, for GPs operating in a mutualistic system - such as Italy dealing autonomously with financial issues requires a major cultural shift, which calls for support from the institutions. In this respect, policy makers could develop policies or actions to push the GPs toward understanding and bearing the economic risk of their actions. In this pathway toward the GPs' awareness, policy makers should spread evidence on which models are providing better results in terms of cost effectiveness (i.e. providing good clinical outcomes, by being economically efficient). This would also meet an expectation raised by some interviewees to generate evidence on best practices within primary care organizations, which could help to legitimize primary care and to limit the risk of failure (a GP argued: "it is not desirable that everyone gets organized anarchically, without any evidence on outcomes: we need someone who has clearer ideas, to pull and stimulate others"). To this end, the definition of performance indicators to orient primary care organizations and the setting of formal meetings for training or for sharing best practices between different groups and networks could be beneficial.

\section{Is Managing a Prerogative for GPs?}

With respect to the framework, despite some differences, we could detect some commonalities in the competences put into practice by the GPs in the organizations they lead. However, some managerial competences such as operations design, data management, negotiation and conflict resolution, are not a prerogative for GPs, but - on the contrary - mostly a prerogative for nurses and assistants. If the policy makers' will in the reform of primary care was to force GPs to organize and manage multi-disciplinary teams, other professionals are supporting the organization and managing the team, while GPs are focusing more on medical practice. As such, policy makers should decide either to implement strategies for empowering GPs on managerial duties, or to invest in coherent training actions for nurses and assistants of primary care organizations (Gradel et al., 2016). 
In this respect, a substantial lack of coherent training, which finds also support in the literature (e.g. Busari et al., 2011; Busari, 2012), emerged from the interviews. GPs do not receive systematic training, neither on managerial competences nor on how to innovate clinical practice through collaboration, but they learn it on the job ("we invented the practice"; "Last week I had a meeting with my colleagues for clinical auditing. I am a tutor for a student at the GPs' residency program, so I ask her to come with me at the meeting, just to show her what it means to work in a group. We were talking about a new project, we really were into how to manage the patients effectively. You wouldn't believe it: she was upset, disoriented. She told me: 'I didn't expect that GPs do things like these. I don't even understand what you are talking about"'). On the other hand, nurses are substantially left apart from any specific training on primary care ("no one told us how to do it: we learn it alone, by doing it again and again"). Assistants, in turn, mentioned that some Local Health Authorities organized courses for them. However, most of them aim to provide updates on the use of the administrative software. Insufficient (or sporadic) training is instead provided on organizational competences ("yeah, we took one of those courses, and we hardly hold back laughing...totally useless and this is a compliment, believe me").

\section{CONCLUSIONS}

Intense debate is ongoing about how to make the primary care systems proactive, bundled and shared. This wave of change requires GPs to adopt a different mindset, from being mostly focused on clinical issues, to become mangers of multi-professional primary care organizations. However, the literature is essentially silent on which competences GPs actually need to manage these new arrangements. In this study, we applied to the Italian context the framework by Bohmer (2010), which collects eight managerial competences for GPs in the new primary care settings (i.e. operations design, data management, human resources management, leadership/negotiation and conflict resolution, team management, innovation and performance measurement, financial control, and capital allocation). Our twofold aim was to understand if all these competences are useful to manage primary care organizations in the practice, and if they are a prerogative for GPs only. To this end, we conducted 16 in-depth interviews to GPs, nurses and assistants. The results showed that the competences included in the framework by Bohmer (2010) emerged as useful to manage the Italian primary care organizations. However, some of them (especially financial control and capital allocation) are still at an immature stage of development. This calls for actions by policy makers, to shape the GPs' mindset and share evidence of best practices. In addition, managerial competences such as operations design, data management, negotiation and conflict resolution, are not a prerogative for GPs, but - on the contrary - mostly a prerogative for nurses and assistants, while GPs are focusing more on innovation the clinical practice. Once again, policy makers should decide either to implement strategies for empowering GPs on organizational duties, or to invest in coherent training actions for nurses and assistants of primary care organizations. 


\section{REFERENCES}

Baron, R. J. (2012). New pathways for primary care: an update on primary care programs from the innovation center at CMS. The Annals of Family Medicine, 10(2), 152-155.

Barzdins, J., \& Barzdins, A. (2013). A pilot study on self-perceived need for management training among medical students in Latvia. International Journal of Medical Education, 4, 59.

Bienkowska-Gibbs, T., King, S., Saunders, C., \& Henham, M. L. (2015). New organisational models of primary care to meet the future needs of the NHS: a brief overview of recent reports. RAND Europe Cambridge.

Blumenthal, D. M., Bernard, K., Bohnen, J., \& Bohmer, R. (2012). Addressing the leadership gap in medicine: residents' need for systematic leadership development training. Academic Medicine, 87(4), 513-522.

Bohmer, R. M. (2010). Managing the new primary care: the new skills that will be needed. Health Affairs, 29(5), 1010-1014.

Busari, J. O., Berkenbosch, L., \& Brouns, J. W. (2011). Physicians as managers of health care delivery and the implications for postgraduate medical training: a literature review. Teaching and learning in medicine, 23(2), 186-196.

Busari, J. (2012). Comparative analysis of quality assurance in health care delivery and higher medical education. Advances in Medical Education and Practice, 3, 121-127.

Chouvarda, I. G., Goulis, D. G., Lambrinoudaki, I., \& Maglaveras, N. (2015). Connected health and integrated care: Toward new models for chronic disease management. Maturitas, 82(1), 22-27.

Cohen, D. J., Davis, M., Balasubramanian, B. A., Gunn, R., Hall, J., Peek, C. J., ... \& Pollack, D. (2015). Integrating behavioral health and primary care: consulting, coordinating and collaborating among professionals. The Journal of the American Board of Family Medicine, 28(Supplement 1), S21S31.

Corbin, J., \& Strauss, A. (2014). Basics of qualitative research: Techniques and procedures for developing grounded theory. Sage publications.

Essex, B., \& Jackson, R. N. (1981). Moneymed: a game to develop management skills in general practice. JR Coll Gen Pract, 31(233), 735-739.

Eyre, H. A., \& Durning, S. (2015). A Measured Approach to Enhancing the Nonclinical Skills of US MDs. Journal of graduate medical education, 7(1), 133-133.

Fitzsimmons, P., \& White, T. (1997). Medicine and management: a conflict facing general practice?. Journal of Management in Medicine, 11(3), 124-131.

Gradel, M., Moder, S., Nicolai, L., Pander, T., Hoppe, B., Pinilla, S., ... \& Dimitriadis, K. (2016). Simulating the physician as healthcare manager: An innovative course to train for the manager role. GMS Journal for Medical Education, 33(3).

Hall, J., Cohen, D. J., Davis, M., Gunn, R., Blount, A., Pollack, D. A., ... \& Miller, B. F. (2015). Preparing the workforce for behavioral health and primary care integration. The Journal of the American Board of Family Medicine, 28(Supplement 1), S41-S51.

Hart, C. K., Dykes, C., Thienprayoon, R., \& Schmit, J. (2015). Change Management in Quality Improvement: The Softer Skills. Current Treatment Options in Pediatrics, 1(4), 372-379.

Homer, C. J., \& Baron, R. J. (2010). How to scale up primary care transformation: what we know and what we need to know?. Journal of general internal medicine, 25(6), 625-629.

Lane, D. S., \& Ross, V. (1998). Defining competencies and performance indicators for physicians in medical management. American journal of preventive medicine, 14(3), 229-236.

Lenander, C., Bondesson, Å., Midlöv, P., \& Viberg, N. (2015). Healthcare system intervention for safer use of medicines in elderly patients in primary care-a qualitative study of the participants' perceptions of self-assessment, peer review, feedback and agreement for change. BMC family practice, 16(1), 1. 
Loeb, D. F., Binswanger, I. A., Candrian, C., \& Bayliss, E. A. (2015). Primary care physician insights into a typology of the complex patient in primary care. The Annals of Family Medicine, 13(5), 451-455.

Margolius, D., \& Bodenheimer, T. (2010). Transforming primary care: from past practice to the practice of the future. Health Affairs, 29(5), 779-784.

Morrin, L., Britten, J., Davachi, S., \& Knight, H. (2013). Alberta Healthy Living Program-A Model for Successful Integration of Chronic Disease Management Services. Canadian journal of diabetes, 37(4), 254-259.

Morrissey, J. (2013). Physicians and New Care Models. Health progress (Saint Louis, Mo.), 95(3), 8-13.

Rubin, G., Berendsen, A., Crawford, S. M., Dommett, R., Earle, C., Emery, J., ... \& Hamilton, W. (2015). The expanding role of primary care in cancer control. The Lancet Oncology, 16(12), 1231-1272.

Saba, G. W., Villela, T. J., Chen, E., Hammer, H., \& Bodenheimer, T. (2012). The myth of the lone physician: toward a collaborative alternative. The Annals of Family Medicine, 10(2), 169-173.

Saltman, R., Bankauskaite, V., \& Vrangbaek, K. (2005). Primary care in the driver's seat?: Organizational reform in European primary care (Vol. 51). McGraw-Hill Education (UK).

Scherger, J. E. (2007). Preparing the personal physician for practice (P4): essential skills for new family physicians and how residency programs may provide them. The Journal of the American Board of Family Medicine, 20(4), 348-355.

Segato, F., \& Masella, C. (2015). Integrated care in action: opening the "black box" of implementation. The International journal of health planning and management.

Strauss, A., \& Corbin, J. (1998). Basics of qualitative research: Techniques and procedures for developing grounded theory. Sage Publications, Inc.

Tallia, A. F., \& Howard, J. (2012). An academic health center sees both challenges and enabling forces as it creates an accountable care organization. Health affairs, 31(11), 2388-2394.

Truglio, J., Graziano, M., Vedanthan, R., Hahn, S., Rios, C., Hendel-Paterson, B., \& Ripp, J. (2012). Global health and primary care: increasing burden of chronic diseases and need for integrated training. Mount Sinai Journal of Medicine: A Journal of Translational and Personalized Medicine, 79(4), 464-474. 